Noname manuscript No.

(will be inserted by the editor)

\title{
The proof by 1 to $n$
}

\section{Questioning the Hodgkin-Huxley model}

\author{
Bernard Delalande \\ Hirohisa Tamagawa \\ Vladimir Matveev
}

Received: date / Accepted: date

\begin{abstract}
The cellular model we teach and have theorized assumes that the cell is the basic unit of multicellular living beings. This fundamental element has been the subject of many theories concerning its properties and the exchanges that exist with its environment.

In this article, we demonstrate that certain functional aspects, in particular the electrical aspects related to diffusion, have not been correctly assumed or that certain initial conditions have been purely ignored and are in contradiction with physics, chemistry and thermodynamics.
\end{abstract}

Keywords cell model · Bernstein · Nernst equation · membrane potential · GHK equation $\cdot \mathrm{HH}$ model

\section{Introduction}

Developing a robust science model is often a difficult project. Many aspects must be taken into account and tons of questions must be answered in a logical and reasoned way. Each cell and even the bacteria have a membrane potential that can be measured between both sides of the membrane. This membrane potential is generally negative by convention. This means that the inside of the

Bernard Delalande

280, avenue de la Pierre Dourdant, 38290 La Verpilliere, France

E-mail: bernard@somasimple.com

Hirohisa Tamagawa

Department of Mechanical Engineering, Faculty of Engineering, Gifu University, 1-1 Yanagido, Gifu, Gifu, 501-1193 Japan

E-mail: tmgwhrhs@gifu-u.ac.jp

Vladimir Matveev

Laboratory of Cell Physiology, Institute of Cytology, Russian Academy of Sciences, Tikhoretsky Ave 4, St. Petersburg 194064, Russia

E-mail: vladimir.matveev@gmail.com 
cell is considered more negative than the outside. The existence and especially the generation of this membrane potential was at the origin of the famous dispute between Volta and Galvani in the $18^{\text {th }}$ century $[1,2,5]$.

Later, Julius Bernstein [6] at the beginning of the $20^{\text {th }}$ century outlined a new theory on the advice of a friend, Ostwald, and put forward a new hypothesis based on the results and work of the famous chemist Nernst. It seems that many aspects of the model have not yet been adequately answered, as many questions have not even been formulated, logic also seems to have sometimes been discarded.

Table 1 Symbols used in the text

\begin{tabular}{l|l}
\hline Symbols & Definition \\
\hline$C$ & Capacitance \\
$V$ & membrane potential difference \\
$E_{K}$ & Nernst potential for potassium \\
$Q_{\text {out }}$ & External charge imbalance \\
$q_{\text {cell. } i}$ & Internal charge imbalance for cell $i$ \\
$R$ & Universal gas constant \\
$T$ & temperature \\
$F$ & Faraday constant \\
$z$ & number of electrons transferred in the chemical reaction \\
\hline
\end{tabular}

\section{The ionic imbalance}

Bernstein's hypothesis based essentially on potassium is the most basic computation of membrane potential. This point is already open to criticism because this statement does not respond to the possibilities of freeing oneself from electroneutrality and ignores all the other electrolyte components that are also electrically charged.

It uses the Nernst equation [4] but without remembering its initial conditions, which are nevertheless mandatory, [7].

$$
E_{K}=\frac{R T}{z F} \cdot \ln \frac{\left[K^{+}\right]_{o u t}}{\left[K^{+}\right]_{\text {in }}}
$$

Since it is not very easy to obtain the internal concentration of potassium on a living cell, the computation is often extrapolated from another formula that comes from the known laws of electricity;

$$
C=\frac{Q}{V}
$$

But this is not at all acceptable since we then lost all our parameters known as ionic concentrations... The computation and existence of the membrane capacitance can also be the subject of much discussion. The computed load 


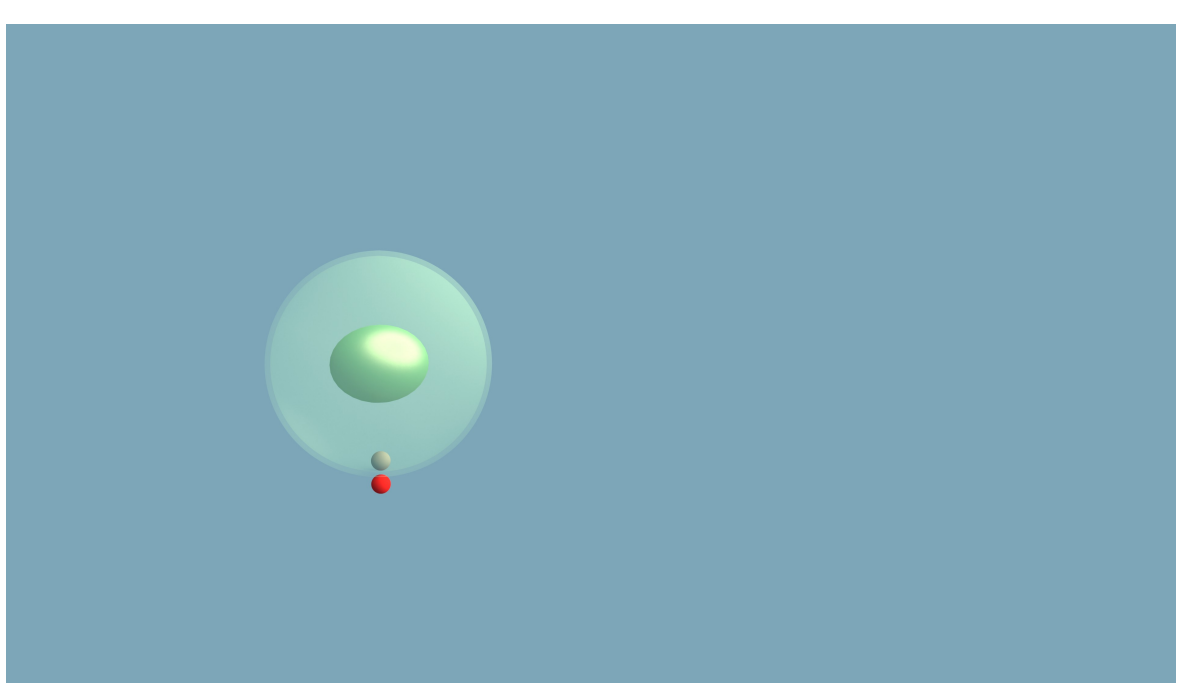

Fig. 1 A single cell from the model used in the hypothesis

$Q$ comes directly from an arbitrary value of the capacitance which is only extrapolated.

Moreover, it is no longer possible to relate the two equations.

It is this $Q$ quantity that would be in excess outside the cell but would remain near the membrane. These ions should be attracted by their counterparts inside the cell by electrostatic forces. This point is also questionable by the cell geometry.

\section{A single but useful model}

Let's take a simple but illustrated model where the imbalance is limited to 1 (and only 1) of elementary charge outside the cell (see fig. 1).

As we assume the membrane can be considered as a capacitance:

- The internal membrane surface holds 1 negative charge.

- The external membrane surface holds 1 positive charge.

The model must work for any type of cell, so each cell has 1 negative charge inside and only 1 positive charge outside. The external environment is generally accepted as being everywhere at the same potential, but it is obvious that it contains the cell itself. Here is our first sample:

The capacitor has equal but opposite charges, which is perfectly acceptable and in accordance with the knowledge we have of it. The whole remains neutral, but each subsystem has a minor imbalance of 1 elementary charge.

We can write;

$$
\begin{gathered}
Q_{\text {out }}+\sum q_{\text {cell.i }}=0 \\
Q_{\text {out }}=\left|\sum q_{\text {cell. } i}\right|
\end{gathered}
$$




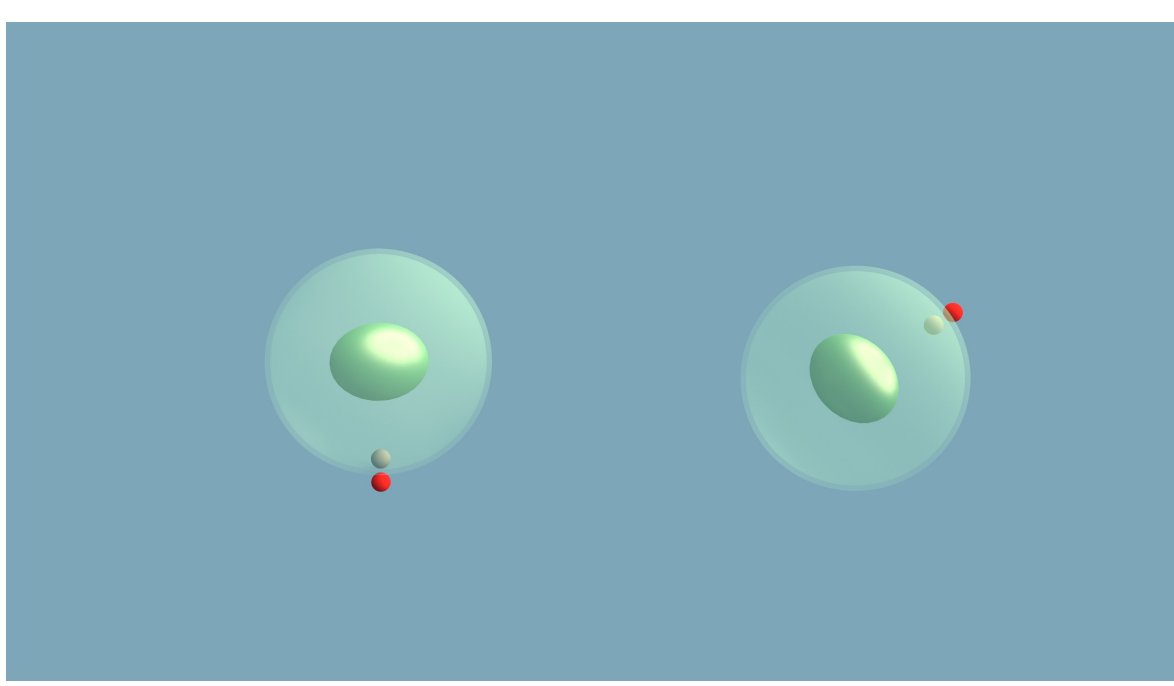

Fig. 2 Two cells from the model. There is already a problem with the capacitance of each cell.

Everything works correctly if you test the model with $\mathrm{i}=1$. But to test its robustness, it is necessary to try to place it in a situation: It is indeed very rare to have a single isolated cell in nature.

\section{Increasing the number towards $n$}

We must test the model when the number of cells is increased; Cells are often organized into tissues and in liquid media the situation is similar. This is a normal situation for living cells or bacteria.

$1 \mathrm{ml}$ of saliva already contains about $75 \cdot 10^{6}$ bacteria.

Let's start again with a smaller number; 2

Here is an image (see fig. 2) of the second trial We can write, again

$$
Q_{\text {out }}+q_{\text {cell.1 }}+q_{\text {cell } .2}=0
$$

But, if the external environment actually contains 2 positive charges, each cell contains only one as expected in our model. Each cell sees the external milieu containing 2 positive ions, but the internal side of the membrane contains only one positive ion.

We are confronted with the first problem of the model: an asymmetric capacitor.

There is no such thing.

This is a violation of equation 2 and known principles regarding capacitors. We will always have 1 charge inside but a charge that goes up outside.

If we take $1 \mathrm{ml}$ of saliva the problem becomes crucial. Furthermore, our gut contains $2 \mathrm{~kg}$ of bacteria. It contains around $10^{14}$ living organisms. The 
external milieu would contain a dangerous charge that will kill us in a second. Why is it that we are still unable to highlight this difference of electric charge? The external environment is easily accessible but the samples are unfailingly neutral. Occam's razor doesn't work. Why? Is it so difficult to say that we were wrong?

The model does not function when $i>1$. However, this is the usual case.

\section{Close encounter of the same type}

We have seen that there are problems with a configuration where the ions are outside but near the membrane.

There are others:

Many living beings are unicellular, can move and exchange with their environment. If we accept that ions can stick to the membrane by electrostatic attraction, we must accept that it only works when there is no stronger attraction that can break this situation. This could occur when a cell encounters an ion at a distance closer than the thickness of the membrane.

$$
\text { ion distance }<\text { thickness }
$$

Unfortunately, the probability of such a encounter is $100 \%$. It will also not work when one cell meets another cell, with the same probability. It will also not work when cells form living tissues.

The model does not work in too many perfectly normal situations. The membrane potential cannot be explained by Bernstein's hypothesis. It cannot be applied in the GHK equation, which is a direct [3] variant that is one of the pillars of modern biology and used in the famous Hodgkin and Huxley model.

The cell has potential because it contains charges, but it is unlikely that they will be connected as an electrical circuit. Their simple presence, at these places, inside the membrane, is already sufficient to produce a membrane potential without any problem.

The starting hypothesis is simple. There are no ions in our external environment. It was to simplify the discussion. If we added them, the situation would be even worse for our model which only copies the current one! The cell will have no way of retaining ions outside the membrane.

\section{Conclusion}

We have demonstrated with a simple model that the diffusion that would cause the membrane potential is not a very good candidate.

This Bernstein model, which takes up the work of Nersnt, improved by Goldmann, Hodgkin and Katz, does not take into account the initial conditions imposed by electrochemistry and did not consider the logical obstacles or limitations imposed by a model that did not foresee the presence of other cells. 


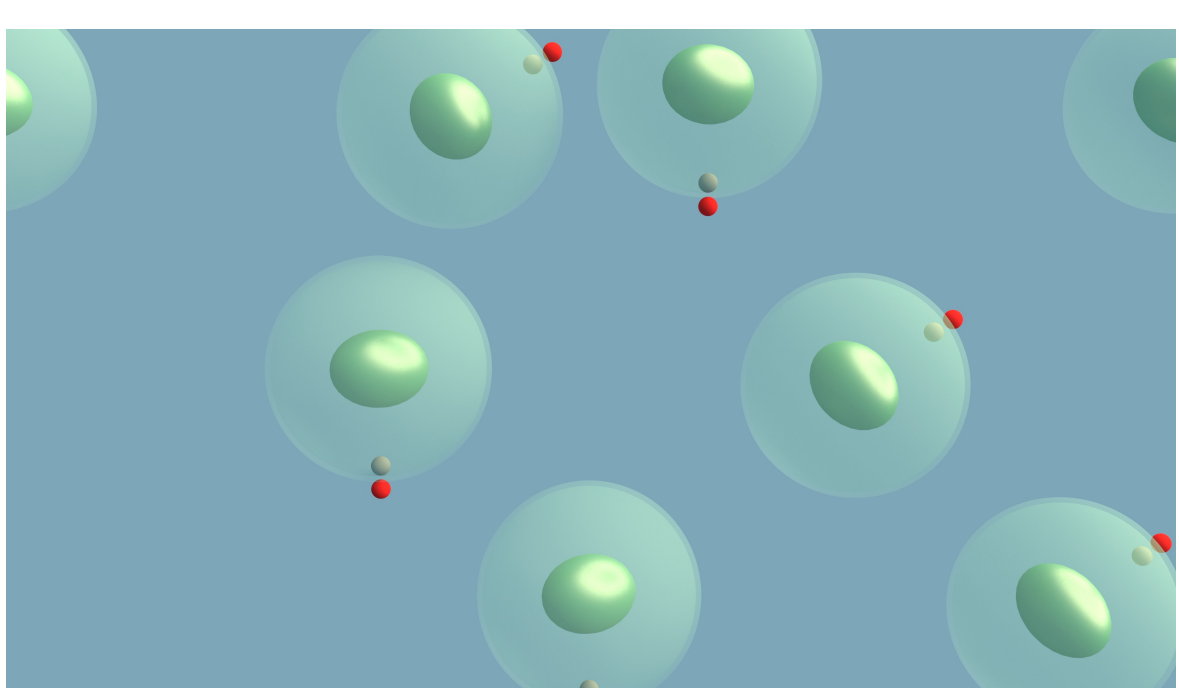

Fig. 3 Some cells from the model. The external system contains too much positive charges and the capacitance of each cell can't work.

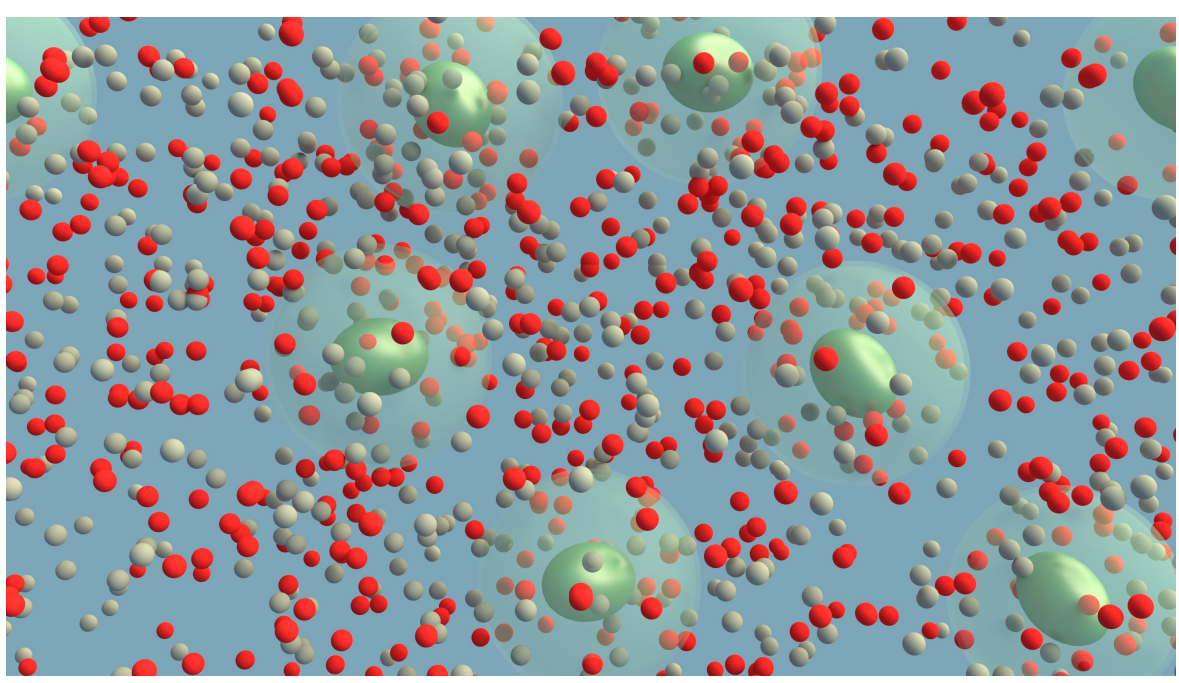

Fig. 4 Some cells from the model. In normal situation the external milieu contains ions. solvent (water molecules) was omitted. Cells can't hold the mandatory ions and ever encounter may ions that break the "system"

\section{References}

1. W. Bernardi. [The controversy over animal electricity in 18th-century Italy: Galvani, Volta, and others]. Revue D'histoire Des Sciences, 54(1):53-70, 2001.

2. Marco Bresadola. Animal electricity at the end of the eighteenth century: the many facets of a great scientific controversy. Journal of the History of the Neurosciences, 17(1):8-32, 2008. 
3. D. E. Goldman. POTENTIAL, IMPEDANCE, AND RECTIFICATION IN MEMBRANES. The Journal of General Physiology, 27(1):37-60, September 1943.

4. Walther Nernst. Begründung der Theoretischen Chemie: Neun Abhandlungen, 18891921. Number Bd. 290 in Ostwalds Klassiker der exakten Wissenschaften. Verlag Harri Deutsch, Frankfurt am Main, 1. aufl edition, 2003. OCLC: ocm52218139.

5. M. Piccolino. Luigi Galvani and animal electricity: two centuries after the foundation of electrophysiology. Trends in Neurosciences, 20(10):443-448, October 1997.

6. Ernst-August Seyfarth. Julius Bernstein (1839-1917): pioneer neurobiologist and biophysicist. Biological Cybernetics, 94(1):2-8, January 2006.

7. Francisco J. Vidal-Iglesias, José Solla-Gullón, Enrique Herrero, Antonio Rodes, and Antonio Aldaz. Do You Really Understand the Electrochemical Nernst Equation? Electrocatalysis, 4(1):1-9, March 2013. 